\title{
Gastrointestinal cancers accompanied by a dense lymphoid component: an overview with special reference to gastric and colonic medullary and lymphoepithelioma-like carcinomas
}

\author{
Runjan Chetty
}

\begin{abstract}
Correspondence to
Professor Runjan Chetty, Department of Cellular Pathology, Oxford University Hospitals/University of Oxford/ Oxford Biomedical Research Centre, Level 1, Academic Centre, John Radcliffe Hospital, Headley Way, Oxford OX3 9DU, UK;

runjan.chetty@ndcls.ox.ac.uk
\end{abstract}

Accepted 26 July 2012 Published Online First 22 August 2012

\begin{abstract}
This review highlights the difference between so-called medullary carcinoma and lymphoepithelioma-like carcinoma with reference to the gastrointestinal tract. The two terms have been applied synonymously in the gastrointestinal tract, with the majority of carcinomas associated with lymphocytes being termed medullary carcinomas. True medullary carcinomas are disposed in syncytial sheets and have well defined peripheral margins; the preponderance of inflammation is peritumoural. These tumours are also associated with microsatellite instability (MSI). Lymphoepithelioma-like carcinomas tend not to have continuous sheets of tumour but are instead made up of small clusters and aggregates of tumour that are broken up by large numbers of intra-tumoural lymphocytes. These tumours may be associated with Epstein-Barr virus (EBV). Occasional cases may be associated with epigenetic silencing of MLH-1. In conclusion, carcinomas associated with dense lymphoid stroma maybe associated with either MSI or EBV. There are some morphological features that favour either type and in the stomach MSI and EBV are mutually exclusive.
\end{abstract}

\section{HISTORICAL ASPECTS AND ORIGINS OF THE TERM 'MEDULLARY CARCINOMA'}

One of the earliest uses of the word 'medullary' in medicine was not as a description of a tumour or some other abnormal condition but rather as a type of normal tissue according to Bichat (General Anatomy Applied to Physiology and Medicine, published in 1801; Professor Juan Rosai, personal communication).

Johannes Muller listed four major types of cancers in 1843: scirrhous, medullary sarcoma, alveolar cancer, and melanotic cancer. Muller's medullary sarcoma was regarded as synonymous with encephaloid cancer and medullary carcinoma (Professor Juan Rosai, personal communication).

The term 'medullary' carcinoma had now entered the medical lexicon on the basis of a variant of breast cancer with microscopic resemblance to the normal medulla oblongata ${ }^{1} 2$ (figure 1). Medullary carcinoma has a low power appearance that highlights the interplay between the inner pale, solid area (corresponding to the cancer) with the surrounding darker zone (corresponding to the lymphoid infiltrate) of the normal medulla oblongata. Some have mistakenly related so-called 'encephaloid' breast cancer with medullary cancer in view of the soft and pale gross appearance that is reminiscent of brain tissue. It would appear that the first description of medullary carcinoma is ascribed to Geschickter, who described a breast cancer with a high survival rate that was accompanied by a dense lymphoid infiltrate. ${ }^{3}$ The actual appellation, 'medullary carcinoma', to describe this unique breast cancer was first used by Foote and Stewart in their classic treatise on the histological classification of breast cancers, and then reinforced three years later by Moore and Foote. ${ }^{12}$ It is interesting to note that even in 1949, Moore and Foote were moved to comment that the word 'medullary', when applied to mammary carcinoma, has been 'variously used by different writers'. ${ }^{2}$

With regard to the gastrointestinal (GI) tract, the first reference to a tumour similar to that occurring in the breast was called a 'blue cell tumour' by Steiner et al in 1948, ${ }^{4}$ but the use of the term medullary carcinoma for an unusual variant of gastric cancer is ascribed to Hamazaki and colleagues, who published this description in the Japanese literature (quoted by Shimizu et al)..$^{5}$ Subsequently, more cases of gastric medullary carcinoma were described, emanating mainly from Japan. ${ }^{7} 8$ Later, cases of medullary carcinoma were also described in the colon. ${ }^{10}$

\section{MORPHOLOGICAL DIAGNOSTIC CRITERIA FOR MEDULLARY CARCINOMA}

In the breast, three systems or sets of criteria were suggested for a histological diagnosis of medullary carcinoma. These were proposed by Ridolfi et al, Wargotz and Silverberg, and Pedersen et al. ${ }^{11-13}$ All three classification schemes are underpinned by common histological features: (a) syncytial growth pattern of the tumour cells in more than $75 \%$ of the tumour, (b) admixed lymphoplasmacytic infiltrate, (c) microscopic circumscription, (d) grade 2 or grade 3 nuclei, and (e) absence of glandular differentiation (figure 2).

In assessing microscopic circumscription of the tumour, it is important to examine the leading edge of the tumour rather than the peri-tumoural lymphocytic response, which may appear slightly irregular. The leading edge of the tumour is non-infiltrative.

Tumours that lack a variable number of these characteristics (depending on the system used) are classified as either atypical medullary carcinoma or invasive ductal carcinoma. The Ridolfi system has the most stringent criteria while the Pedersen system the least stringent. An essential feature is 


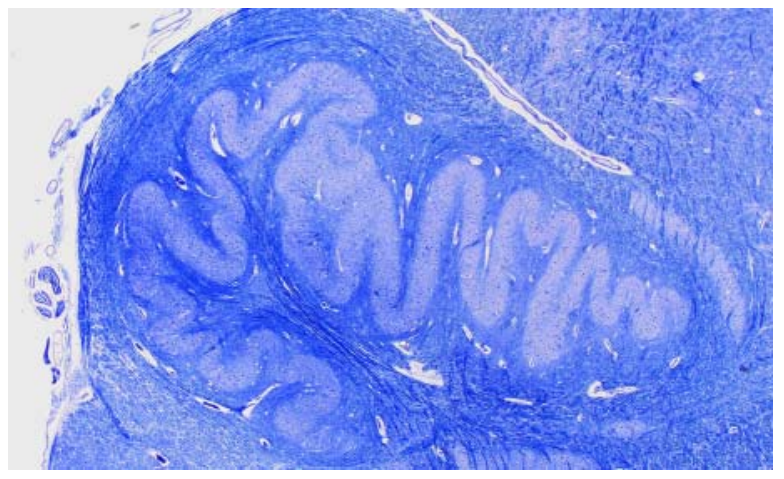

Figure 1 A cross section through the medulla oblongata showing a festooning pale area rimmed by a darker cuff. Medullary carcinomas are thought to resemble this by having syncitia of large cells (light areas) surrounded by a peripheral zone of lymphoid cells (darker areas). (Image courtesy of Dr Olaf Ansorge, Department of Neuropathology, Oxford University Hospitals and University of Oxford.)

the syncytial growth pattern which Ridolfi and colleagues described as 'broad, interanastomosing sheets of tumour cells'. ${ }^{11}$ Wargotz and Silverberg refined this a little by stating the syncytial pattern should be composed of 'broad confluent bands of tumour cells more than five cells thick, often with indistinct cell borders'. ${ }^{12}$ Even the two-criteria system advocated by Pedersen et al, ${ }^{13}$ contained a syncytial growth pattern as one of the two criteria.

\section{GASTROINTESTINAL MEDULLARY CARCINOMA}

The initial descriptions of medullary carcinoma in the stomach and colon have remained faithful to the morphological diagnostic criteria established for their breast counterparts.

Jessurun and colleagues stated quite categorically that 'tumours were accepted as medullary adenocarcinomas if on histological examination at least $80 \%$ showed a solid pattern, had a nested, organoid, or trabecular growth pattern mimicking endocrine carcinomas, and were formed by small round to polygonal uniform cells with scant eosinophilic or amphophilic cytoplasm and rounded nuclei with small central nucleoli'. ${ }^{10}$ They did not include a lymphocytic component as a diagnostic

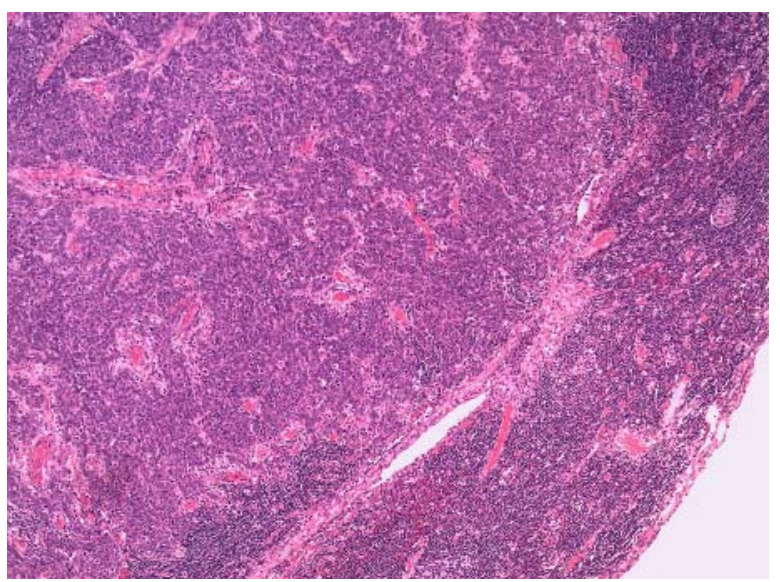

Figure 2 An example of a medullary carcinoma composed of large cells arranged in a diffuse sheet. The edge of the tumour is well defined and regular. The preponderance of lymphocytes is at the periphery of the tumour. Scattered intra-tumoural lymphocytes are also noted. criterion, but all 11 of their cases were associated with a lymphocytic response, which was intense peri- and intratumoural in eight cases. ${ }^{10}$

The next major study on medullary carcinomas of the GI tract was undertaken by Wick and colleagues. ${ }^{14}$ Their selection criteria for medullary carcinoma were as follows: the tumours 'were required to demonstrate sheets, nests, and trabeculae of large polygonal cells with high nuclear/cytoplasmic $(\mathrm{N} / \mathrm{C})$ ratios and amphophilic cytoplasm, with variably interspersed mature lymphocytes. Nuclei were vesicular, with prominent nucleoli'. ${ }^{15}$ This study stressed two important features. First, medullary carcinoma of the colon had a characteristic scanning magnification appearance that is best described as 'organoid', simulating the growth pattern of developing embryonic organs. Second, they emphasised the similarity to neuroendocrine carcinoma morphologically (nested, organoid growth pattern) and immunophenotypically, with over $30 \%$ of their cases showing immunohistochemical evidence of neuroendocrine differentiation. ${ }^{14}$

Thus, an organoid or solid or sheet-like or trabecular pattern, and peri- and intra-tumoural lymphocytes are required to make a diagnosis of medullary carcinoma.

\section{MEDULLARY CARCINOMAS OF THE COLON AND STOMACH AND MICROSATELLITE INSTABILITY}

Medullary carcinomas of the colon became the focus of attention following the discovery of microsatellite instability (MSI) in a cohort of right-sided inherited and sporadic colon cancers. In the setting of Lynch syndrome (hereditary non-polyposis colorectal carcinomas, HNPCC), several of the cancers were noted to be associated with dense Crohn's-like lymphoid aggregates. ${ }^{16}$ In the first detailed pathological examination of sporadic colon cancers showing replicative errors, Kim and colleagues encountered three undifferentiated carcinomas that fulfilled the criteria for medullary carcinomas. ${ }^{15}$ Alexander et $a l^{17}$ also confirmed that a medullary phenotype identifies colorectal cancer with MSI. Thus, a characteristic constellation of morphological features in colon cancer (including colonic medullary carcinoma) was indicative of the molecular status/ pathogenesis of the cancer.

Those medullary carcinomas occurring in the colon have a predilection for the right colon and the characteristic morphology flags a subset of tumours that are microsatellite unstable. A similar association exists between similar appearing medullary gastric cancers and MSI. ${ }^{18}$

\section{EPSTEIN-BARR VIRUS-ASSOCIATED GASTRIC CANCER ('LYMPHOEPITHELIOMA-LIKE CANCER')}

At this juncture it is worth noting that no mention is made of an association between true medullary carcinoma (in any site, including the stomach) and Epstein-Barr virus (EBV). Specifically addressing the question whether EBV was implicated in medullary breast cancer, two studies demonstrated clearly that there was no connection between bona fide medullary breast cancer and EBV. ${ }^{19} 20$

The term 'lymphoepithelioma' was coined in 1921 by Regaud and Reverchon ${ }^{21}$ and Schminke ${ }^{22}$ to describe a tumour occurring in the nasopharynx. This tumour characteristically is composed of cells with large vesicular nuclei, single prominent nucleoli and indistinct cell borders, which impart a syncytial appearance to the tumour, and it has an attendant lymphoplasmacytic inflammatory component. ${ }^{23}$ Following from these seminal observations, two histological patterns are apparent. In the Regaud pattern the tumour is arranged in nests, while in 
the Schminke pattern isolated tumour cells within the characteristic inflammatory infiltrate are encountered. With this phenotypic hallmark, lymphoepithelioma-like carcinomas outside the nasopharynx were soon described: thymus, skin, uterine cervix, lung, salivary gland, breast etc., but the vast majority of these cases were not associated with EBV infection.

Burke et $a l^{23}$ first described a 'lymphoepithelial carcinoma' of the stomach that was associated with EBV. Then in 1991, two studies from the same group described undifferentiated gastric carcinoma that was associated with $\mathrm{EBV}^{24} 25$ In both of these papers the authors clearly refer to the cancers as being 'lymphoepithelioma-like carcinomas' because they bore a resemblance to nasopharyngeal carcinoma, both histologically and because of the EBV association.

Lymphoepithelioma-like carcinomas of the breast that have been described in the literature thus far have not demonstrated an association with $\mathrm{EBV}^{26-30}$

It is worth reiterating that unlike medullary carcinomas, these lymphoepithelioma-like carcinomas in the breast were characterised by tumour cells forming small clusters, nests or sheet-like growth patterns with unclear margins because they were permeated by numerous lymphocytes (figure 3). These findings do not correspond to the syncytial growth patterns seen in medullary carcinoma. Furthermore, the lymphoid infiltrate tended to be more intra-tumoural than peri-tumoural in lymphoepithelioma-like carcinoma of the breast and other sites. In medullary carcinomas, the converse is true.

Thus, in the stomach, carcinomas with a lymphoid stroma are either associated with MSI (medullary carcinoma) or EBV (lymphoepithelioma-like carcinoma). As mentioned, there are morphological features that allow for their separation.

In 1994, a study by Takano et a ${ }^{31}$ was published under the title, 'Epstein-Barr-virus-associated medullary carcinomas with lymphoid infiltration of the stomach'. However, scrutiny of the image and accompanying legend tends to suggest that the tumour depicted is a lymphoepithelioma-like carcinoma rather than a medullary carcinoma. ${ }^{31}$ The tumour that is illustrated has a prominent intra-tumoural lymphocytic response. Furthermore, 'gland formation, usually in acinar or trabecular fashion' was noted in 18 of the 30 cases. It is quite conceivable that some of the cases in the series were, in fact, not medullary carcinomas but EBV-driven lymphoepithelioma-like carcinomas since $90 \%$ of their cases were Epstein Barr encoded RNA-in situ hybridisation (EBER-ISH) positive. ${ }^{31}$

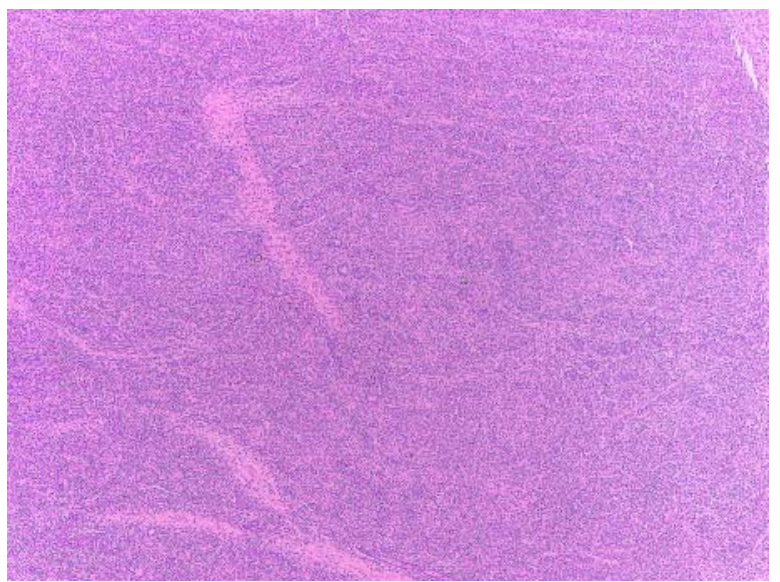

Figure 3 Lymphoepithelioma-like carcinoma is often composed of small clusters of tumour with a pervasive intra-tumoural lymphoid response. The edge of the tumour is infiltrative and irregular.

\section{MSI AND EBV IN GASTRIC CANCERS WITH LYMPHOID STROMA}

Grogg and colleagues explored the relationship between EBV and MSI in the setting of lymphocyte-rich gastric cancers. ${ }^{32}$ Although their numbers were small, an interesting conclusion emanating from their study was that EBV and MSI were mutually exclusive. In other words, none of the tumours that were EBV-positive were MSI-high. ${ }^{32}$ Similar findings were obtained in the study by Leung et al. ${ }^{33}$

These studies lend credence to the notion that there are two separate pathways involved in the genesis of medullary carcinomas and lymphoepithelioma-like carcinomas of the stomach. Although they are united by the presence of lymphoid cells, there are other morphological and pathogenetic differences that allow for their separation. In the stomach it appears safe to assume that not all gastric cancers with a lymphoid stroma are medullary carcinomas. Indeed, if such a cancer is not MSI high, it will more than likely be EBV-positive, and vice versa. The morphology also faithfully recapitulates these different pathogenetic lines: medullary carcinomas being organoid, syncytial and sheet-like with a pushing non-infiltrative tumour front with a dense peri-tumoural lymphocytic infiltrate, while lymphoepithelioma-like carcinoma is composed of single cells, small clusters of cells and small glands with an infiltrative growth pattern and a dense intra-tumoural lymphoid infiltrate.

\section{LYMPHOEPITHELIOMA-LIKE CARCINOMA OF THE COLON}

Thus far, only six cases of lymphoepithelial lesion (LEL) carcinoma have been documented in the colon and the association with EBV has been inconsistent. ${ }^{34-39}$ In fact, the majority of cases, as with several examples of lymphoepithelioma-like carcinoma outside of the nasopharynx/stomach (non-foregut examples), are not associated with EBV. A case of lymphoepithelioma-like carcinoma of the colon has been described in a patient with HNPCC. ${ }^{36}$ From the description of the tumour, it would appear to conform to the criteria for a lymphoepithelioma-like carcinoma rather than a medullary carcinoma. ${ }^{36}$ The patient had additional features such as high-grade tubular adenomas and a mucinous carcinoma. Furthermore, because of the lymphoepithelioma-like morphology, EBV infection was sought and found to be negative. ${ }^{36}$ This does appear to be a unique case and others have not found significant levels of MSI in lymphoepithelioma-like carcinomas of the lung, for instance. ${ }^{40}$ There are cases of colonic lymphoepithelioma-like carcinomas of the colon that are not arranged in syncytial sheets but rather as single cells, small clusters of tumour and occasional glands that have a predominant intra-tumoural lymphoid component. Such a case was EBV negative but showed loss of MLH1 and PMS2 proteins and was deemed to be due to epigenetic loss of these proteins. ${ }^{39}$

\section{CONCLUSIONS}

As the molecular pathways involved in colorectal carcinogenesis are unravelled, the prevailing wisdom is that three distinct pathways exist: the chromosomal instability, MSI and the CpG island methylator phenotype pathways. The latter two are associated with cancers that have a prominent lymphoid component. Lymphoepithelioma-like cancer, if not related to EBV, then may be due to sporadic epigenetic silencing of MLH-1.

As with many terms that are entrenched in medicine, contemporary advances have rendered them somewhat obsolete. Perhaps it would be simpler to classify a tumour with a prominent intra- or peri-tumoural lymphoid component as an 
adenocarcinoma with lymphoid stroma with or without MSI, or with or without associated EBV. There is persuasive evidence that MSI and EBV infection are mutually exclusive, especially in gastric adenocarcinomas with a lymphoid stroma. There is merit in separating the two entities because of their different aetiopathogenesis, genetic screening and potential therapeutic implications.

\section{Take-home messages}

- There is strong molecular evidence that supports separating medullary carcinomas from lymphoepithelioma-like cancers.

- All cancers accompanied by significant lymphocytes that occur in the colon should not simply be regarded as medullary carcinomas.

- Both medullary and lymphoepithelioma-like carcinomas of the gastrointestinal tract have slightly different morphological criteria and are underpinned by distinct molecular pathways.

- Medullary carcinomas are usually associated with MSI, while lymphoepithelioma-like carcinomas may or may not be Epstein-Barr virus-driven.

\section{Competing interests None.}

Provenance and peer review Not commissioned; internally peer reviewed.

\section{REFERENCES}

1. Foote $\mathbf{F W} \mathbf{~ J r}$, Stewart FW. A histologic classification of carcinoma of the breast. Surgery 1946;19:74-99.

2. Moore $\mathbf{O S} \mathbf{J r}$, Foote FW Jr. The relatively favorable prognosis of medullary carcinoma of the breast. Cancer 1949;2:635-43.

3. Geschickter CF. Diseases of the breast; diagnosis, pathology, treatment. Philadelphia: JB Lippincott Co., 1945.

4. Steiner PE, Maimon SN, Palmer WL, et al. Gastric cancer morphologic factors in five-year survival after gastrectomy. Am J Pathol 1948;24:947-61.

5. Hamazaki M, Sawayama K, Kuriya T. Stomach cancer with lymphoid stroma. Saibokakubyourigaku Zasshi 1968;12:115-20 (in Japanese).

6. Shimizu K, Tkaiyama W, Mandai K, et al. Undifferentiated carcinoma with lymphoid infiltration of the esophagus: a case report. Jpn J Clin Oncol 1999;29:494-7.

7. Watanabe H, Enjoji M, Imai T. Gastric carcinoma with lymphoid stroma: its morphologic characteristics and prognostic correlations. Cancer 1976;38:232-43.

8. Kaibara N, Kimura 0 , Nishidoi $\mathrm{H}$, et al. High incidence of liver metastasis in gastric cancer with medullary growth pattern. J Surg Oncol 1985;28:195-8.

9. Jessurun J, Romero-Guadarrama M, Manivel JC. Cecal poorly differentiated adenocarcinoma, medullary type. Mod Pathol 1992;5:43A.

10. Jessurun J, Romero-Guadarrama M, Manivel JC. Medullary adenocarcinoma of the colon: clinicopathologic study of 11 cases. Hum Pathol 1999;30:843-8.

11. Ridolfi RL, Rosen PP, Port A, et al. Medullary carcinoma of the breast: a clinicopathologic study with 10 year follow-up. Cancer 1977;40:1365-85.

12. Wargotz ES, Silverberg SG. Medullary carcinoma of the breast: a clinicopathologic study with appraisal of current diagnostic criteria. Hum Pathol 1988;19:1340-6.

13. Pedersen L, Zedeler K, Holck S, et al. Medullary carcinoma of the breast, proposal for a new simplified histopathological definition. Based on prognostic observations and observations on inter- and intraobserver variability of 11 histopathological characteristics in 131 breast carcinomas with medullary features. Br J Cancer 1991;63:591-5.

14. Wick MR, Vitsky JL, Ritter JH, et al. Sporadic medullary carcinoma of the colon. A clinicopathologic comparison with nonhereditary poorly differentiated enteric-type adenocarcinoma and neuroendocrine colorectal carcinoma. Am J Clin Pathol 2005; 123:56-65.

15. Kim H, Jen J, Vogelstein B, et al. Clinical and pathological charcateristics of sporadic colorectal carcinomas with DNA replication errors in microsatellite sequences. Am J Pathol 1994;145:148-56

16. Smyrk TC, Lynch HT, Watson PA, et al. Histologic features of hereditary nonpolyposis colorectal carcinoma. In: Utsunomiya J, HT Lynch, eds. Hereditary colorectal cancer. Tokyo: Springer-Verlag, 1990:357-62.

17. Alexander $\mathbf{J}$, Watanabe $T, W u T$, et al. Histopathological identification of colon cancer with microsatellite instability. Am J Pathol 2001;158:527-35.

18. Lu B-J, Lai M, Cheng L, et al. Gastric medullary carcinoma, a distinct entity associated with microsatellite instability- $\mathrm{H}$, prominent intraepithelial lymphocytes and improved prognosis. Histopathology 2004;45:485-92.

19. Gaffey MJ, Frierson HF, Mills SE, et al. Medullary carcinoma of the breast: identification of lymphocyte subpopulations and their significance. Mod Pathol 1993;6:721-8.

20. Lespagnard L, Cochaux P, Larsimont D, et al. Absence of Epstein-Barr virus in medullary carcinoma of the breast as demonstrated by immunophenotyping, in situ hybridization and polymerase chain reaction. Am J Clin Pathol 1995;103:449-52.

21. Regaud C, Reverchon L. Sur un cas d'epitheliome epidermoide developpe dams les massif maxillaire superieur. Rev Laryngol Otol Rhinol 1921;42:369-78.

22. Schminke A. Uber lympho-epitheliale Geschwulste. Beitr Pathol Anat Allg Pathol 1921;68:161-70

23. Burke AP, Yen TSB, Shekitka KM, et al. Lymphoepithelial carcinoma of the stomach with Epstein-Barr virus demonstrated by polymerase chain reaction. Mod Pathol 1990;3:377-80.

24. Weiss LM, Gaffey MJ, Shibata D. Lymphoepithelioma-like carcinoma and its relationship to Epstein-Barr virus. Am J Clin Pathol 1991;96:156-8.

25. Shibata D, Tokunaga M, Uemura Y, et al. Association of Epstein-Barr virus with undifferentiated gastric carcinomas with intense lymphoid infiltration. Am J Pathol 1991;139:469-74.

26. Kumar S, Kumar D. Lymphoepithelioma-like carcinoma of the breast. Mod Pathol 1994; 7:129-31.

27. Cristina $\mathbf{S}$, Boldorini R, Brustia F, et al. Lymphoepithelioma-like carcinoma of the breast: an unusual pattern of infiltrating lobular carcinoma. Virchows Arch 2000;437:198-202.

28. Naidoo P, Chetty R. Lymphoepithelioma-like carcinoma of the breast with associated sclerosing lymphocytic lobulitis. Arch Pathol Lab Med 2001;125:669-72.

29. Dadmanesh F, Peterse JL, Sapino A, et al. Lymphoepithelioma-like carcinoma of the breast: lack of evidence of Epstein-Barr virus infection. Histopathology 2001;38:54-61.

30. Kurose A, Ichinohasama R, Kanno $\mathrm{H}$, et al. Lymphoepithelioma-like carcinoma of the breast. Report of a case with the first electron microscopic study and review of the literature. Virchows Arch 2005;447:653-9.

31. Takano $\mathbf{Y}$, Kato $\mathbf{Y}$, Sugano $\mathrm{H}$. Epstein-Barr-virus-associated medullary carcinomas with lymphoid infiltration of the stomach. J Cancer Res Clin Oncol 1994;120:303-8.

32. Grogg KL, Lohse CM, Pankratz $\mathrm{S}$, et al. Lymphocyte-rich gastric cancer: associations with Epstein-Barr virus, microsatellite instability, histology, and survival. Mod Pathol 2003;16:641-51.

33. Leung SY, Yuen ST, Chung LP, et al. Microsatellite instability, Epstein-Barr virus, mutation of type $\|$ transforming growth factor $\beta$ receptor and BAX in gastric carcinoma in Hong Kong Chinese. Br J Cancer 1999;79:582-8.

34. Vilor M, Tsutsumi Y. Localization of Epstein-Barr virus genome in lymphoid cells in poorly differentiated adenocarcinoma with lymphoid stroma of the colon. Pathol Int 1995; $45: 695-7$

35. Samaha S, Tawfik 0, Horvat R, et al. Lymphoepithelioma-like carcinoma of the colon. Dis Colon Rectum 1998;41:925-8.

36. De Petris G, Lev R, Quirk DM, et al. Lymphoepithelioma-like carcinoma of the colon in a patient with hereditary nonpolyposis colorectal cancer. Arch Pathol Lab Med 1999;123:720-4.

37. Kon S, Kasia K, Tsuzuki N, et al. Lymphoepithelioma-like carcinoma of the rectum: possible relation with EBV. Pathol Res Prac 2001;197:577-82.

38. Kojima Y, Mogaki M, Takagawa R, et al. A case of lymphoepithelioma-like carcinoma of the colon with ulcerative colitis. J Gastroenterol 2007;42:181-5.

39. Chetty R, Delaney D. Lymphoepithelioma-like carcinoma of the colon. Int J Clin Exp Pathol 2012;5:105-9.

40. Dacic S, Lomago D, Hunt JL, et al. Microsatellite instability is uncommon in lymphoepithelioma-like carcinoma of the lung. Am J Clin Pathol 2007;127:282-6. 